\title{
MISCELANEA
}

PRIMERAS JORNADAS CIENTÍFICAS DE ESTUDIANTES DE ENFERMERÍA. FACULTAD CIENCIAS DE LA SALUD. UNIVERSITAT JAUME I. CASTELLÓN DE LA PLANA.

\section{DIAGNóstico DE SALUd COMUNITARIA DE ALCANAR}

Autores: I. Ros Cardo, I. Llagostera Reverter, A. Ventura Pérez, C. Pitarch Albado, N. Roda Julio; Salas Medina P.

Correo electrónico: al242006@uji.es

Institución: Universitat Jaume I.

Tipo de comunicación: oral.

Introducción: El diagnostico de salud es el resultado de un estudio epidemiológico de una comunidad, a partir de una descripción y análisis de las necesidades de salud. Es decir, es la medición del estado de salud de la población en un momento determinado mediante indicadores de salud. 
Objetivos: Generales: Analizar la situación de salud e identificar las necesidades poblacionales reconocidas.

Secundarios: Identificar los elementos necesario que permitan la elaboración del diagnóstico de salud de Alcanar. Conocer los recursos que dispone el municipio para satisfacer las necesidades poblacionales.

Material y métodos: Estudio descriptivo transversal. La recogida de datos se realizó en distintas instituciones de la población y de las bases de datos estadísticas de la Generalitat de Catalunya. Se realizó una revisión de la literatura con el fin de localizar la mayor evidencia relacionada con las actividades planteadas.

Resultados: Los resultados en los indicadores de niveles de salud, de condiciones sanitarias, de recursos y actividades, indicadores económicosociales, educativos y socio-económicos, de educación, del estado nutricional de la población, sobre saneamiento y urbanismo, son óptimos. Las enfermedades de más prevalencia son la HTA, obesidad, DMT2, seguidas del Alzheimer con $1.76 \%$.

Discusión: La necesidad pertinente de intervención fue la atención a los enfermos de Alzheimer, no por ser la enfermedad con más prevalencia, sino por no presentar un plan de intervención, porque afecta tanto al paciente como al cuidador y ha aumentado su incidencia. Las intervenciones van dirigidas a disminuir el deterioro cognitivo, físico y aliviar los problemas conductuales. 